\title{
Non-wetting droplets in capillaries of circular cross-section: Scaling function
}

Cite as: Phys. Fluids 31, 043102 (2019); https://doi.org/10.1063/1.5087722

Submitted: 03 January 2019 . Accepted: 13 March 2019 . Published Online: 03 April 2019

Karol Makuch (D), Jean-Baptiste Gorce (D), and Piotr Garstecki (D)
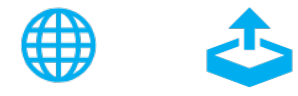

\section{ARTICLES YOU MAY BE INTERESTED IN}

Formation of water in oil in water particles by drop impact on an oil layer

Physics of Fluids 31, 037107 (2019); https://doi.org/10.1063/1.5089001

Computational analysis of self-similar capillary-driven thinning and pinch-off dynamics during dripping using the volume-of-fluid method

Physics of Fluids 31, 021211 (2019); https://doi.org/10.1063/1.5061715

Influence of electric field on deformation of a drop in shear flow

Physics of Fluids 31, 042102 (2019); https://doi.org/10.1063/1.5087066

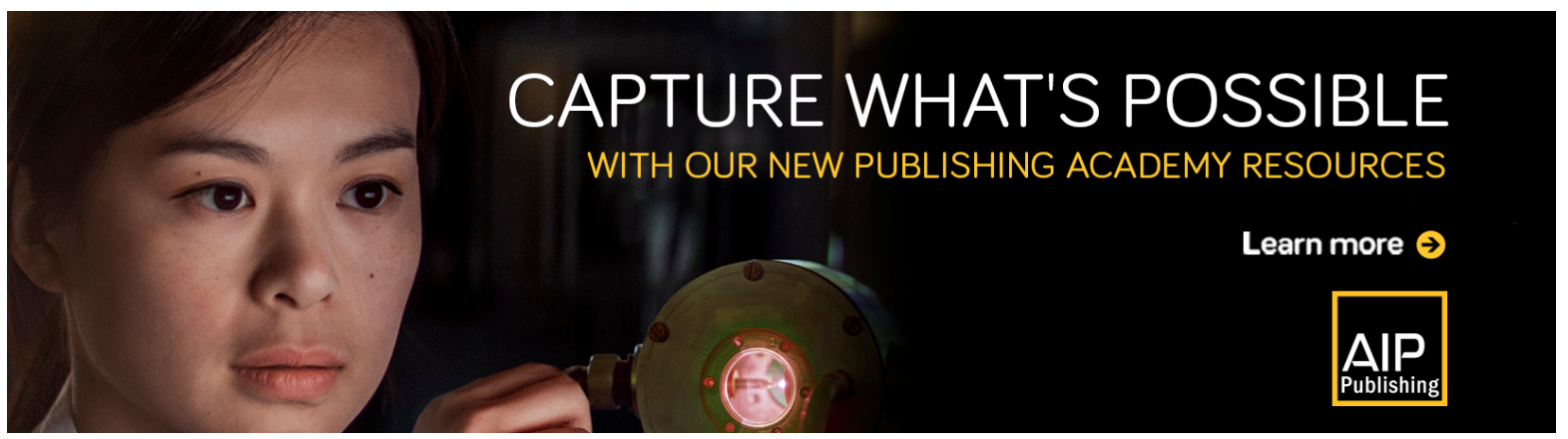




\title{
Non-wetting droplets in capillaries of circular cross-section: Scaling function
}

\author{
Cite as: Phys. Fluids 31, 043102 (2019); doi: 10.1063/1.5087722 \\ Submitted: 3 January 2019 - Accepted: 13 March 2019 • \\ Published Online: 3 April 2019
}

\author{
Karol Makuch, ${ }^{1}$ (D) Jean-Baptiste Gorce, ${ }^{2}$ (D) and Piotr Garstecki \\ AFFILIATIONS \\ ${ }^{1}$ Institute of Physical Chemistry, Polish Academy of Sciences, Kasprzaka 44/52, 01-224 Warsaw, Poland \\ ${ }^{2}$ Research School of Physics and Engineering, The Australian National University, Canberra ACT 2601, Australia
}

\begin{abstract}
a) Author to whom correspondence should be addressed: garst@ichf.edu.pl
\end{abstract}

\begin{abstract}
Steady motion of long, non-wetting droplets carried by a surrounding liquid in a circular capillary has been the subject of many experimental, theoretical, and numerical simulation studies. Theoretical approaches, even after the application of lubrication approximation in hydrodynamic equations and after neglecting inertia and gravity effects, still lead to a numerical procedure to determine the speed of a droplet or the thickness of the film between a droplet and the wall of the capillary. Here, we develop the lubrication approximation further to introduce an analytical formula for the speed of droplets as a function of the capillary number and of the ratio of the viscosity coefficients of the two immiscible phases. We achieve this by identification of a scaling function within the lubrication approximation. The equations that we propose here corroborate well with the results of numerical simulations of droplet flow in circular capillaries.
\end{abstract}

Published under license by AIP Publishing. https://doi.org/10.1063/1.5087722

\section{INTRODUCTION}

We consider the stationary motion of a long, non-wetting viscous droplet in a tube of circular cross section. The practical motivation for the interest in the speed of droplets is fueled by the dynamic development and use of the techniques of droplet microfluidics. Droplets are used in biological experiments to cultivate microorganisms ${ }^{1}$ and in chemical experiments as reactors. ${ }^{2}$ The ability to link the mobility (or speed) of droplets with the material parameters of their content could allow the development of label free methods to infer properties of droplets from their speeds, as, for example, to judge the density of a bacterial culture inside the droplet from the measurement of its speed.

In this article, we describe an intermediate step to achieve this goal by introducing an algebraic formula for the thickness of the film between a droplet and a capillary and for the speed of a long, nonwetting droplet in a channel of circular cross section.

Fairbrother and Stubbs were one of the first who raised the question whether the speed of the flow of a gas bubble moving in a tube filled with a liquid may serve as a proxy for the speed of the liquid itself. ${ }^{4}$ The speed of a long, non-wetting droplet which flows in a steady motion in a tube also differs from the average speed of the surrounding (continuous) liquid that wets the walls of the tubing. Between the droplet and the wall, there is a thin wetting film of the continuous liquid. As a result, the cross section swept by the droplet is smaller than the lumen of the tube (Fig. 1). We study the mobility $\beta$ of the droplet defined by the ratio of the speed of the droplet $U$ to the average speed of the continuous phase

$$
\beta=\frac{U}{V} \text {. }
$$

Fairbrother and Stubbs observed that the length of a bubble $l$ does not affect its mobility, when the length is larger than three tube radii, $l>3 r$. A theoretical expression of the mobility of a bubble has been introduced by Bretherton. ${ }^{6}$ To find the mobility of a bubble, Bretherton used Stokes equations with surface tension on the liquid's interface. In the region where the profile of the interface is almost parallel to the channel's wall, Bretherton neglected perpendicular components of the velocity (lubrication approximation). He also considered no-slip boundary conditions on both the interfacesbetween the droplet and the continuous liquid and between the continuous phase and the walls of the channel. This leads to an ordinary differential equation for the profile of the film-i.e., the thickness of the film along the length of the droplet. Bretherton also used the fact that at small values of the capillary number, the front of the bubble has a hemi-spherical shape. By matching the curvature of the profile of the film with the curvature of a semi-spherical cap in a small-slope region, he was able to calculate the film thickness, $\epsilon \equiv b / r$, 


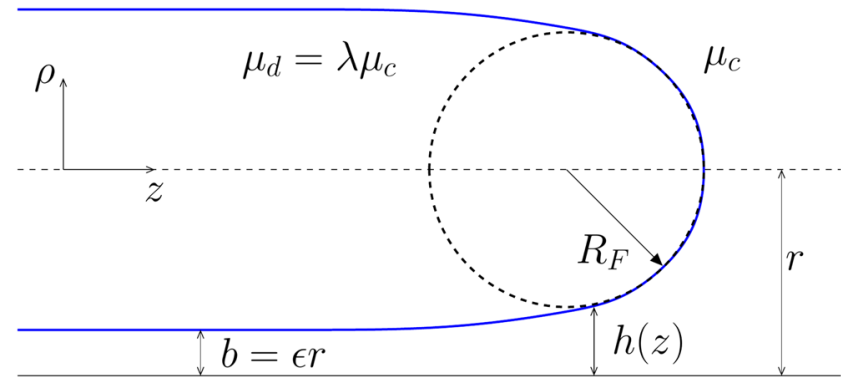

FIG. 1. Schematic sketch of the droplet moving in a circular tube.

as a function of the capillary number

$$
\epsilon=0.643(3 C a)^{\frac{2}{3}} \text {. }
$$

Bretherton also derived the formula for the mobility which we represent by

$$
\beta=1+1.29(3 \mathrm{Ca})^{\frac{2}{3}} .
$$

The film thickness and the mobility of a bubble are determined solely by the capillary number $C a=\left(U \mu_{c}\right) / \sigma$, which contains the viscosity of the continuous phase, $\mu_{c}$, and the surface tension, $\sigma$. Bretherton obtained the above formula for a droplet with zero viscosity, $\mu_{d}=0$, in a tube in the limit of small capillary numbers by neglecting the effects of inertia in hydrodynamic equations, neglecting also gravity effects, and assuming uniform distribution of the surface tension.

Bretherton's approach has later been extended to the case of a viscous droplet. Finite viscosity of the liquid inside the droplet requires taking into account viscous forces exerted by the droplet phase on the surrounding continuous liquid. The procedure can be found, e.g., in the papers of Schwartz et al. ${ }^{7}$ and Teletzke et al., and it is also described in detail in the recent paper of Balestra et al. ${ }^{9}$ Within the lubrication approximation, Bretherton neglected the components of the velocity and pressure field in Stokes equations which are perpendicular to the tube axis. He used this assumption in the layer between the droplet and the wall of the channel. In the case of a viscous droplet, lubrication approximation is extended for the velocity field inside the droplet. ${ }^{7-9}$ For a viscous droplet, formula (2) generalizes to the following equation:

$$
\epsilon=(3 C a)^{\frac{2}{3}} P(\lambda \epsilon)
$$

[cf. Eq. (3.18) in Ref. 7], with function $P(m)$ which Schwartz et al. determined numerically. For a given viscosity ratio $\lambda$ and the capillary number $\mathrm{Ca}$, one must solve numerically Eq. (4) to calculate the film thickness, $\epsilon$. Knowing the film thickness, one can determine the mobility of a long viscous droplet from the formula of Goldsmith and Mason

$$
\beta=\frac{1+\left(2 \epsilon-\epsilon^{2}\right)(-1+2 \lambda)}{1+\left(4 \epsilon-6 \epsilon^{2}+4 \epsilon^{3}-\epsilon^{4}\right)(-1+\lambda)} .
$$

According to our knowledge, the above procedure is the state of the art, i.e., the simplest method to calculate the film thickness around a long non-wetting droplet and its speeds in the limit of small capillary numbers within the lubrication approximation. Equation (4) cannot be solved analytically, as it requires iterative numerical approximations. Here, we show that further analysis within the lubrication approximation for viscous droplets is possible.

First, we identify a scaling equation for the film thickness. Second, we derive algebraic formulas to determine the mobility of a long, non-wetting droplet in terms of the capillary number and the contrast of viscosity coefficients between the droplet and continuous liquids.

\section{RESULTS}

The starting point for the derivation is formula (4). It has three independent parameters $\epsilon, \lambda$, and $C a$. When used in such a form, it must be solved numerically for each pair of values of $C a$ and $\lambda$. Here, we show that the notation can be simplified to avoid the need for numerical solutions. We multiply both sides of Eq. (4) by the viscosity ratio $\lambda$, obtaining $\lambda \epsilon=\lambda(3 C a)^{\frac{2}{3}} P(\lambda \epsilon)$. The resulting equation can be expressed in terms of only two independent parameters

$$
m \equiv \lambda \epsilon
$$

and

$$
g \equiv \lambda(3 C a)^{\frac{2}{3}} .
$$

This substitution simplifies formula (4) to the following equation $m=g P(m)$. Solution of this equation defines $m$ as a function of $g$, which we represent in the following form:

$$
m=g M(g) \text {. }
$$

We determine $M(g)$ numerically and introduce the following fitting formula for our numerical solution:

$$
\begin{gathered}
M(g) \approx M_{f i t}(g)=b_{0}+\frac{g+b_{4} g^{2}+\left(b_{0} 2^{2 / 3}-b_{0}\right) g^{3}}{b_{1}+b_{2} g+b_{3} g^{2}+g^{3}}, \\
b_{0}=0.643, b_{1}=4.109, b_{2}=8.906, b_{3}=10.144, b_{4}=3.575 .
\end{gathered}
$$

Figure 2 shows the function $M_{f i t}(g)$. The relative error of the fit in comparison with the numerically exact result, $\left|M(g)-M_{f i t}(g)\right| /$ $M(g)$, is less than $4 \times 10^{-3}$ for the whole range of $g \in(0, \infty)$, as shown in Fig. 3.

Combination of Eqs. (6)-(8) leads to the following expression:

$$
\epsilon=(3 \mathrm{Ca})^{\frac{2}{3}} M\left(\lambda(3 \mathrm{Ca})^{\frac{2}{3}}\right),
$$

and with the fit given by formula (9), it determines the film thickness. Along with formula (5), the above equation also determines the mobility of droplets. For the case of inviscid droplets, $\lambda=0$, we recover Bretherton's results for the film thickness and mobility given by Eqs. (2) and (3), respectively.

Importantly, the film thickness in Eq. (10) is represented by a single parameter function, $M(g)$. It follows that the combination of the film thickness and capillary number $\epsilon /(3 \mathrm{Ca})^{\frac{2}{3}}$ collapses into a single curve, $\epsilon /(3 \mathrm{Ca})^{\frac{2}{3}}=M\left(\lambda(3 \mathrm{Ca})^{\frac{2}{3}}\right)$ described by the $M(g)$ function. For this reason, we call $M(g)$ the scaling function.

Algebraic formulas (5), (9), and (10) for the film thickness and mobility of droplets contain the capillary number, $\mathrm{Ca}=U \mu_{c} / \sigma$, based on the speed of droplets. It is also interesting to know the form of those formulas in terms of capillary numbers based on the speed 


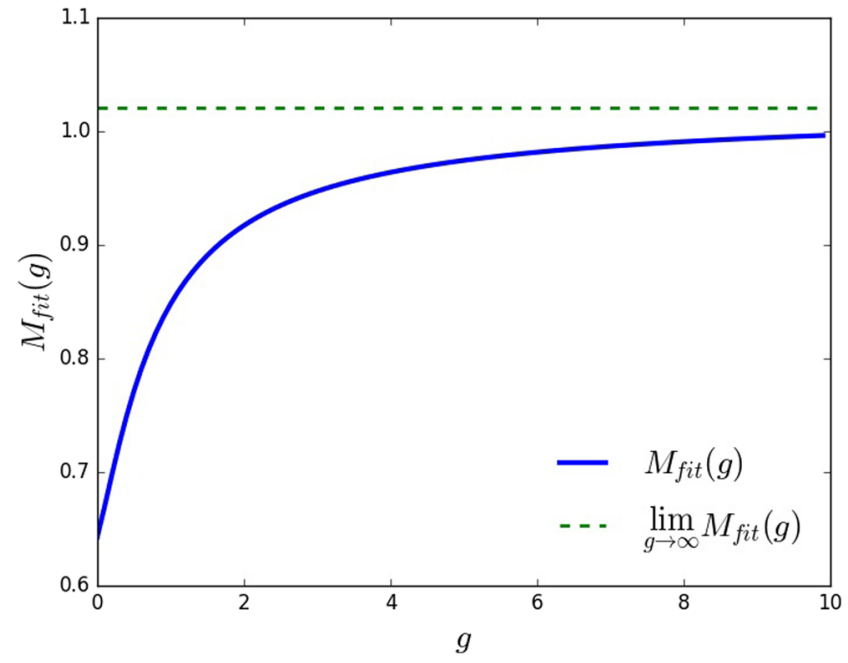

FIG. 2. Scaling function $M_{\text {fit }}(g)$ from Eq. (9).

of the continuous phase, $C a_{c}=V \mu_{c} / \sigma$. Both capillary numbers are related by the mobility, $\mathrm{Ca}=C a_{c} \beta$. Using this relation in Eqs. (5), (9), and (10), one can obtain expressions for the film thickness and mobility in terms of $\mathrm{Ca}_{c}$. To the leading order for small capillary numbers, those expressions are the same as Eqs. (5), (9), and (10), but with $\mathrm{Ca}_{c}$ instead of $\mathrm{Ca}$. The difference between using capillary numbers based on the speed of droplets and the speed of the continuous phase in Eqs. (5), (9), and (10) is of the order of $\epsilon^{2}$ for the film thickness and the mobility.

Figure 4 compares the film thickness obtained by our procedure with the results of numerical simulations. Among different available results, ${ }^{10-15}$ we use the results by Balestra et al. ${ }^{9}$ who performed numerical simulations for a wide range of capillary numbers including the careful study of capillary numbers around $10^{-3}$. In the limit of small capillary numbers, we expect a perfect agreement

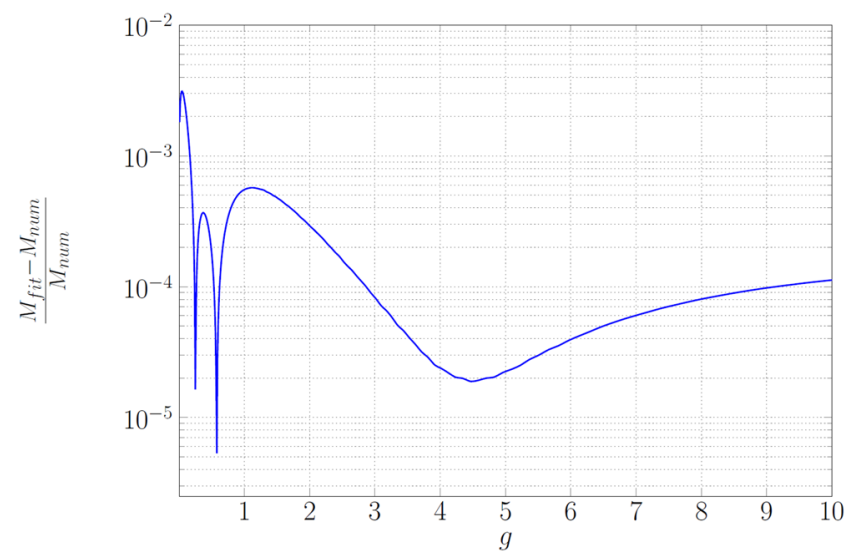

FIG. 3. Difference between the scaling function determined numerically using the method described by Schwartz et al. ${ }^{7}$ denoted by $M_{\text {num }}(g)$ and its fit $M_{\text {fit }}(g)$ from Eq. (9).

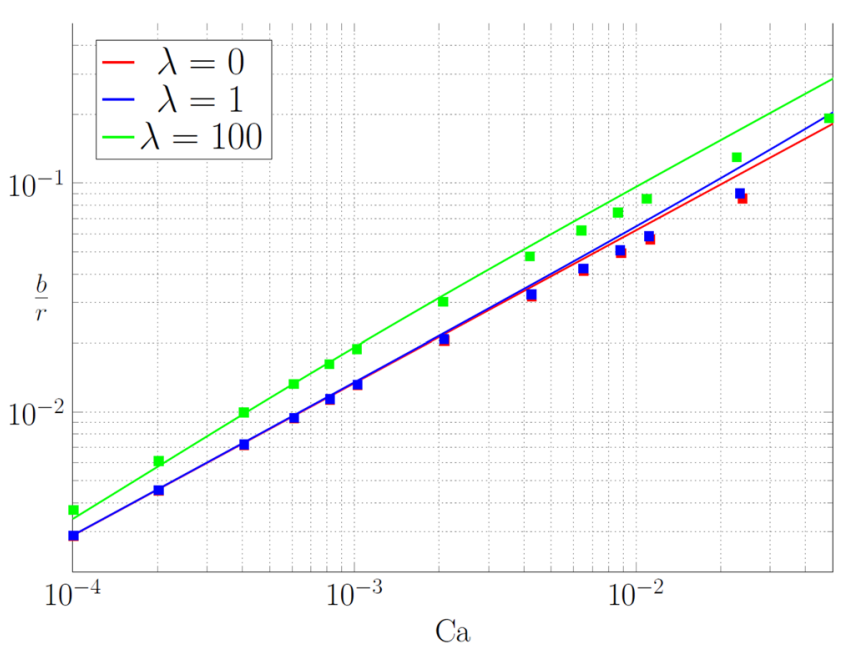

FIG. 4. Film thickness between the wall and the droplet as a function of the capillary number $\mathrm{Ca}$ for different viscosity ratios $\lambda=0,1$, and 100 . The lines represent lubrication approximation given by Eqs. (9) and (10). The squares represent the numerical simulations from Balestra et al.

between the numerical and analytical results because the approximations used to derive our formulas are expected to work in this regime. Consequently, for higher capillary numbers, the analytical results should start deviating from numerical results. This picture is confirmed by the comparison presented in Fig. 4. The comparison for viscosity ratios $\lambda=0,1,100$ shows that the lubrication approximation described by Eqs. (9) and (10) follows numerical simulations. In both cases, the film thickness increases with the capillary number and decreases with the viscosity ratio. The difference between the lubrication approximation and the numerical simulations increases with the capillary number, but it would be possible to recognize the

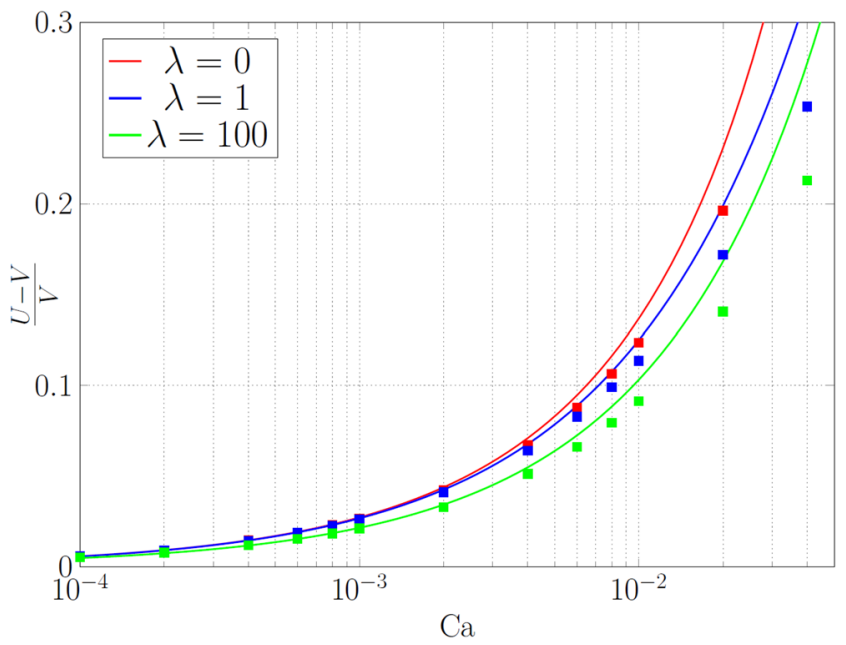

FIG. 5. Droplet's mobility as a function of the capillary number $\mathrm{Ca}$ for different viscosity ratios $\lambda=0,1$, and 100 . The lines represent lubrication approximation given by Eqs. (5), (9), and (10). The squares represent the numerical simulations from Balestra et al. 
difference between droplets with $\lambda=0$ and $\lambda=100$ for capillary numbers up to $C a \approx 10^{-2}$. For example, in case of water droplets in hexadecane, this value corresponds to the speed $0.8 \mathrm{~m} / \mathrm{s}$. We found similar agreement between the numerical simulations and the lubrication approximation for the case of speeds of viscous droplets. The comparison is presented in Fig. 5. Up to $C a \approx 10^{-2}$, the difference between numerical and our theoretical values, $\left|\beta-\beta_{\text {sim }}\right| /\left(\beta_{\text {sim }}-1\right)$, is less than $10 \%$.

\section{DISCUSSION}

The key point of this article is identification of the scaling function for the film thickness in the lubrication approximation for long, non-wetting droplets. This is expressed by passing from Eq. (4) to expression (10), and it allows the introduction of algebraic formulas (5), (9), and (10) for the film thickness and mobility of droplets. In perspective, it can be used to infer properties of a droplet from its speed in microfluidic experiments, such as recent very precise measurements of the film thickness ${ }^{16}$ and speed of droplets for capillary numbers below $10^{-3}$. 3 .17 The speed of the droplet requires measuring distance and time, which can be done very precisely. For example, Sklodowska et al. ${ }^{3}$ measured time of passage between two sensors with the relative accuracy about $10^{-4}$ for capillary number $C a \approx 10^{-4}$. Answering the question how precisely the viscosity (or surface tension) can be inferred from the lubrication approximation with our algebraic formulas demands further study. There is also an open question whether the above concept of scaling function can be applied in the case of channels of different cross sections, in approaches going beyond lubrication approximations such as Hodges et al.,${ }^{18}$ in extensions for higher capillary numbers, ${ }^{19-21}$ or in determination of pressure drop induced by the droplet. 9,22

\section{ACKNOWLEDGMENTS}

We are grateful to Gioele Balestra for data of numerical simulations published in Ref. 9. K.M. has received funding from the National Science Center, Poland (No. 2016/21/D/ST3/00988). P.G. acknowledges support from the Polish National Science Centre based on Decision No. DEC-2014/12/W/NZ6/00454 (Symfonia).

\section{REFERENCES}

${ }^{1}$ T. S. Kaminski, O. Scheler, and P. Garstecki, "Droplet microfluidics for microbiology: Techniques, applications and challenges," Lab Chip 16(12), 2168-2187 (2016).

${ }^{2}$ K. S. Elvira, X. C. i Solvas, R. C. R. Wootton et al., “The past, present and potential for microfluidic reactor technology in chemical synthesis," Nat. Chem. 5(11), 905915 (2013).
${ }^{3}$ K. Sklodowska, P. R. Debski, J. A. Michalski, P. M. Korczyk, M. Dolata, M. Zajac, and S. Jakiela, "Simultaneous measurement of viscosity and optical density of bacterial growth and death in a microdroplet," Micromachines 9(5), 251 (2018).

${ }^{4}$ F. Fairbrother and A. E. Stubbs, "Studies in electro-endosmosis. Part VI. The bubble-tube method of measurement," J. Chem. Soc. (Resumed) 1935, 527-529.

${ }^{5} \mathrm{H}$. L. Goldsmith and S. G. Mason, "The flow of suspensions through tubes. II. Single large bubbles," J. Colloid Sci. 18(3), 237-261 (1963).

${ }^{6}$ F. P. Bretherton, "The motion of long bubbles in tubes," J. Fluid Mech. 10(02), 166-188 (1961).

${ }^{7}$ L. W. Schwartz, H. M. Princen, and A. D. Kiss, "On the motion of bubbles in capillary tubes,” J. Fluid Mech. 172, 259-275 (1986).

${ }^{8}$ G. F. Teletzke, H. T. Davis, and L. E. Scriven, "Wetting hydrodynamics," Rev. Phys. Appl. 23(6), 989-1007 (1988).

${ }^{9}$ G. Balestra, L. Zhu, and F. Gallaire, "Viscous Taylor droplets in axisymmetric and planar tubes: From Bretherton's theory to empirical models," Microfluid. Nanofluid. 22(6), 67 (2018).

${ }^{10}$ E. Lac and J. D. Sherwood, "Motion of a drop along the centreline of a capillary in a pressure-driven flow," J. Fluid Mech. 640, 27-54 (2009).

${ }^{11}$ M. J. Martinez and K. S. Udell, "Axisymmetric creeping motion of drops through circular tubes," J. Fluid Mech. 210, 565-591 (1990).

${ }^{12}$ E. J. Soares and R. L. Thompson, "Flow regimes for the immiscible liquid-liquid displacement in capillary tubes with complete wetting of the displaced liquid," J. Fluid Mech. 641, 63-84 (2009).

${ }^{13}$ E. J. Soares, M. S. Carvalho, and P. R. Souza Mendes, "Immiscible liquid-liquid displacement in capillary tubes," J. Fluids Eng. 127(1), 24-31 (2005).

${ }^{14} \mathrm{H}$. Westborg and O. Hassager, "Creeping motion of long bubbles and drops in capillary tubes," J. Colloid Interface Sci. 133(1), 135-147 (1989).

${ }^{15}$ T. M. Tsai and M. J. Miksis, "Dynamics of a drop in a constricted capillary tube," J. Fluid Mech. 274, 197-217 (1994).

${ }^{16}$ A. Huerre, O. Theodoly, A. M. Leshansky, M.-P. Valignat, I. Cantat, and M.-C. Jullien, "Droplets in microchannels: Dynamical properties of the lubrication film," Phys. Rev. Lett. 115(6), 064501 (2015).

${ }^{17}$ D. Ferraro, M. Serra, D. Filippi, L. Zago, E. Guglielmin, M. Pierno, S. Descroix, J.-L. Viovy, and G. Mistura, "Controlling the distance of highly confined droplets in a capillary by interfacial tension for merging on-demand," Lab Chip 19(1), 136-146 (2019)

${ }^{18}$ S. R. Hodges, O. E. Jensen, and J. M. Rallison, "The motion of a viscous drop through a cylindrical tube," J. Fluid Mech. 501, 279-301 (2004).

${ }^{19} \mathrm{P}$. Aussillous and D. Quéré, "Quick deposition of a fluid on the wall of a tube," Phys. Fluids 12(10), 2367-2371 (2000).

${ }^{20}$ E. Klaseboer, R. Gupta, and R. Manica, "An extended Bretherton model for long Taylor bubbles at moderate capillary numbers," Phys. Fluids 26(3), 032107 (2014).

${ }^{21}$ J. A. Howard and P. A. Walsh, "Review and extensions to film thickness and relative bubble drift velocity prediction methods in laminar Taylor or slug flows," Int. J. Multiphase Flow 55, 32-42 (2013).

${ }^{22}$ A. Cherukumudi, E. Klaseboer, S. A. Khan, and R. Manica, "Prediction of the shape and pressure drop of Taylor bubbles in circular tubes," Microfluid. Nanofluid. 19(5), 1221-1233 (2015). 